\title{
EXPERIMENTAL STUDIES OF THE NASOPHARYNGEAL SECRETIONS FROM INFLUENZA PATIENTS.
}

\section{ImMunity Reactions.}

By PETER K. OLITSKY, M.D., AND FREDERICK L. GATES, M.D.

(From the Laboratories of The Rockefeller Institute for Medical Research.)

(Received for publication, July 16, 1921.)

In the earlier experiments of this series in which the anaerobic, filter-passing microorganism, Bacterium pneumosintes, was transmitted from the nasopharyngeal secretions of influenza patients to the respiratory tract of rabbits, and passed from rabbit to rabbit through many generations, it was our practice to inoculate two or more rabbits in each experiment. One or more of these animals was killed after 24 to 48 hours, as already described, ${ }^{1}$ for study and to obtain material for further inoculation. The infection, however, was permitted to run its course in a number of rabbits, which upon recovery became available for subsequent studies in immunity.

The experiments made with the latter rabbits may be grouped according to the nature of the injected material (Table I). These animals were reinjected after varying intervals with material from other sources. The tests were particularly useful in establishing the identity of the active agent derived from human nasopharyngeal washings, and from the lung tissues of affected rabbits, with the cultures of Bacterium pneumosintes.

A single typical protocol of the experiments will serve to show the method followed and the results obtained.

Group A. Influenzal Nasopharyngeal Washings versus Active Agent in Lungs of Affected Rabbits.

Experiment 1.-Rabbit $A$ for 4 days prior to injection showed an average leucocyte count of 11,821 , of which 6,190 were mononuclears, and temperature of $39.6^{\circ} \mathrm{C}$. Mar. 29,1919 . Inoculated intratracheally with $3 \mathrm{cc}$. of the unfiltered nasopharyngeal washings from Case 16.1 Mar. 30. Conjunctivitis. Total

${ }^{1}$ Olitsky, P. K., and Gates, F. L., J. Exp. Med., 1921, xxxiii, 125. 
leucocytes 9,000 , of which 3,600 were mononuclears. Temperature $39.6^{\circ} \mathrm{C}$. Mar. 31. Conjunctivitis. Total leucocytes 7,025, of which 1,967 were mononuclears. Temperature $40^{\circ} \mathrm{C}$. Apr. 1. Conjunctivitis. Total leucocytes 6,325, of which 2,404 were mononuclears. Temperature $40^{\circ} \mathrm{C}$. Apr. 2. Recovered. Total leucocytes 11,500 , of which 4,140 were mononuclears. Temperature $39.4^{\circ} \mathrm{C}$. The animal remained well until Apr. 21, 1919, when it was reinjected, intratracheally, with $3 \mathrm{cc}$. of lung tissue suspension from a rabbit representing the fifth passage of the nasopharyngeal secretions from Case 17. ${ }^{1}$ This active material had been filtered between the third and fourth rabbit passages. No observable effects on the rabbit's appearance, behavior, blood count, or temperature resulted.

As a control for the first injection, Rabbit B was injected along with Rabbit A, and with the same material. For 2 days after the injection Rabbit B showed a

TABLE I.

Groups of Immunity Experiments.

\begin{tabular}{|c|c|c|}
\hline Group. & First injection. & Second injection. \\
\hline $\mathbf{A}$ & $\begin{array}{l}\text { Nasopharyngeal washings from influ- } \\
\text { enza patients. }\end{array}$ & $\begin{array}{l}\text { Suspensions of lungs of affected } \\
\text { rabbits. }\end{array}$ \\
\hline B & Suspensions of lungs of affected rabbits. & $\begin{array}{l}\text { Similar suspensions from another } \\
\text { animal. }\end{array}$ \\
\hline $\mathrm{C}$ & $\begin{array}{l}\text { Washed mass cultures of Bacterium } \\
\text { pneumosintes. }\end{array}$ & Similar cultures of another strain. \\
\hline $\mathbf{D}$ & Suspensions of lungs of affected rabbits. & $\begin{array}{l}\text { Washed mass cultures of Bacterium } \\
\text { pneumosinies. }\end{array}$ \\
\hline $\mathbf{E}$ & $\begin{array}{l}\text { Washed mass cultures of Bacterium } \\
\text { pneumosintes. }\end{array}$ & $\begin{array}{l}\text { Suspensions of lungs of affected } \\
\text { rabbits. }\end{array}$ \\
\hline
\end{tabular}

typical clinical picture, with a fall in the leucocyte count, affecting especially the mononuclears, and a rise in temperature. The animal was then killed and autopsy revealed the characteristic hemorrhagic edema and emphysema of the lungs, without involvement of other organs. ${ }^{1}$ Similarly, Rabbit C served as a control for the second injection of Rabbit A, and showed typical clinical and pathological effects.

This experiment, typical of three in this series, indicates the development of a protective resistance to the active agent in the rabbit passage experiments, as the result of the previous injection of the nasopharyngeal secretions from an early case of influenza. The nasopharyngeal secretions are thus further shown to be the source of the filterable active material of the rabbit passages. In this instance, 
the interval between injections was 23 days. In another experiment, the resistance was maintained for 3 months.

\section{Group B. Active Agent in Lungs of Affected Rabbits versus Similar Material Originally Derived from Other Patients.}

That the strains of the active agent in lungs of affected rabbits, originally derived from different influenza patients, have the same antigenic properties and thus give rise to protection against each other is brought out by the following experiment.

Experiment 2.-Rabbit A, having a normal leucocyte count of 14,525 , of which 6,100 were mononuclears, and a temperature of $39.8^{\circ} \mathrm{C}$., was injected intratracheally on Feb. 25, 1919, with 3 cc. of a saline solution suspension of rabbit lung from the fourth passage of the nasopharyngeal secretions of Case 11,1 obtained during the first influenzal wave of 1918-19. The subsequent train of events carried the mononuclear cells to 1,242 , and the temperature to $40.65^{\circ} \mathrm{C}$. on the 3 rd day. The fever and mononuclear leucopenia persisted on the 4 th and 5 th days, with recovery on the 6 th and 7 th days, when the total leucocyte count was 20,450 , of which 5,521 were mononuclears, and temperature $39.6^{\circ} \mathrm{C}$. Nearly 14 months later, May 11, 1920, this animal was injected with $3 \mathrm{cc}$. of a suspension of glycerolated lung tissue from the first rabbit inoculated with the nasopharyngeal secretions of Case 26, from the second or 1920 epidemic. The rabbit developed no significant changes in the blood count or temperature, and when killed, on May 13 , showed no visible lung lesions.

The control rabbit, B, similarly injected with the same glycerolated lung tissue, developed the typical blood picture and lung lesions, and the presence of the active agent in the lungs was demonstrated by transmission to a second rabbit which reacted in the characteristic manner.

This experiment not only demonstrates the identity of the antigenic characters of the active material derived from the two epidemics of 1918-19 and of 1920, but shows that the immunity conferred by a previous infection with the active agent may last for a long time.

With the isolation of Bacterium pneumosintes from the nasopharyngeal secretions of influenza patients and from the lung tissue of affected rabbits, it became possible to study the character of the active agent of the animal passages by means of cross-protection experiments. It was first necessary to show that recovery from infection with Bacterium pneumosintes induces resistance to a subsequent inoculation of the same microorganism. 


\section{Group C. Bacterium pneumosintes versus the Same Microorganism.}

Experiment 3.-Rabbit A, normal leucocyte count 9,600, of which 6,144 were mononuclears. Temperature $39.3^{\circ} \mathrm{C}$. Injected intratracheally with $3 \mathrm{cc}$. of a saline suspension of washed mass culture in the seventh generation of a rabbit lung strain of Bacterium pneumosintes from Case $16,{ }^{1}$ a patient from the first (1918-19) epidemic wave. 1st day after injection: total leucocytes 8,400 , of which 2,520 were mononuclears; temperature $39.3^{\circ} \mathrm{C}$. 2nd day: total leucocytes 3,800 , of which 1,444 were mononuclears; temperature $39.6^{\circ} \mathrm{C}$. 3rd day: total leucocytes 5,400 , of which 2,052 were mononuclears; temperature $39.6^{\circ} \mathrm{C}$. 4th day: recovery; total leucocytes 11,600 , of which 4,640 were mononuclears; temperature $39.3^{\circ} \mathrm{C}$. 5th day: total leucocytes 15,000 , of which 7,500 were mononuclears; temperature $39.4^{\circ} \mathrm{C}$. On the following day, when the total leucocytes were 13,600 , of which 5,440 were mononuclears, and the temperature was $39.8^{\circ} \mathrm{C}$., this animai was reinjected with a similar washed mass culture of a strain of Bacterium pneumosintes in the seventh generation from the filtered nasopharyngeal secretions of Case 17,1 from the same epidemic. During the next 5 days the total leucocyte count remained about 13,000, with an increase in the mononuclear cells to an average of 8,292 , and the temperature was constant at $39.7^{\circ} \mathrm{C}$.

For assurance that the injected mass culture was active and capable of infecting normal rabbits, a control animal was injected, each time, along with Rabbit A, and with the same material. For 48 hours after injection, these rabbits, B and C, showed the characteristic leucocytic depression, involving especially the mononuclears. In each instance they were then killed, and the usual emphysema and hemorrhagic edema of the lungs were found..$^{2}$

To show that the reactions described were due to Bacterium pneumosintes and not to some constituent of the culture medium, Rabbit D was inoculated intratracheally with $3 \mathrm{cc}$. of a saline suspension of the sediment of uninoculated, sterile, mass culture medium, at the time that Rabbit A was first injected. The animal gave no reaction. At the time of the second injection, Rabbit $D$ was also injected with the seventeenth generation of the culture of the nasopharyngeal secretions of Case 17. This animal promptly developed the clinical and pathological syndrome characteristic of infection with Bacterium pneumosintes. ${ }^{2}$

\section{Group D. Active Agent in Lungs of Affected Rabbits versus Bacterium pneumosintes.}

We have already stated" that "the active material, pathogenic for rabbits and guinea pigs, found in the nasopharyngeal secretions of patients in the early hours of uncomplicated epidemic influenza has been identified in the anaerobic organism" (Bacterium pneumosintes).

${ }^{2}$ Olitsky, P. K., and Gates, F. L., J. Exp. Med., 1921, xxxiii, 713. 
Additional evidence in support of this statement is furnished by the results of cross-protection experiments of which the following are examples.

Experiment 4.-Rabbit A, normal leucocyte count, 9,200, of which 6,072 were mononuclears. Temperature $39^{\circ} \mathrm{C}$. Injected intratracheally with $3 \mathrm{cc}$. of a saline solution suspension of rabbit lung tissue representing the fifth passage of the filtered nasopharyngeal secretions from Case 26. ${ }^{1}$ The following day, leucocytes were 6,400 , of which 2,560 were mononuclears. Temperature $39.3^{\circ} \mathrm{C}$. 2nd day: leucocytes 7,200 , of which 2,763 were mononuclears; temperature $39.4^{\circ} \mathrm{C}$. 3rd day: leucocytes 4,400 , of which 1,760 were mononuclears; temperature $39.4^{\circ} \mathrm{C}$. 4th day: recovery with leucocytes 11,000 , of which 6,600 were mononuclears; temperature $39.2^{\circ} \mathrm{C}$. 15 days later this rabbit was injected intratracheally with $3 \mathrm{cc}$. of a saline solution suspension of washed mass culture of Bacterinm pneumosintes, second generation, originally derived from the nasopharyngeal secretions of the same patient. On the 2 days following, the total leucocyte counts were 14,600 and 16,800 , of which 7,800 and 9,240 were mononuclears; temperature $39.5^{\circ} \mathrm{C}$. Killed. Autopsy revealed no visible lesions in the lungs. The activity of the materials used in these injections was demonstrated by the injection of control rabbits, $B$ and $C$, both of which gave typical reactions. In similar experiments, the reinjected rabbits were observed for several days after the second injection to detect any delayed effects. They remained normal.

In Experiment 4, the interval between the first and second injections was 15 days. In three other experiments, identical results were obtained after 3 weeks, and in a fifth, after 9 weeks. In a sixth experiment, involving an interval of 18 days, the second injection produced the typical clinical and pathological reaction. That such protection experiments should occasionally be unsuccessful is not surprising, in view of the arbitrary dosage employed.

\section{Group E. Bacterium pneumosintes versus Active Agent in Lungs of Affected Rabbits.}

The converse of Experiment 4 was performed in a series in which Bacterium pneumosintes was injected first and the animals were later exposed to the influenzal active agent by the intratracheal injection of affected rabbit lung.

Experiment 5.-Rabbit A showed a normal leucocyte count of 12,000, of which 6,240 were mononuclears. Temperature $39^{\circ} \mathrm{C}$. It was injected intratracheally with $3 \mathrm{cc}$. of a saline solution suspension of washed sediment from a mass culture 
of Bacterium pneumosintes. During the following 3 days, the total leucocyte count averaged 7,200, of which 2,200 were mononuclears, and the temperature rose to $39.6^{\circ} \mathrm{C}$. Thereafter the animal returned to normal. 2 weeks after the first injection, this rabbit was inoculated intratracheally with $3 \mathrm{cc}$. of a suspension of the active agent in rabbit lung tissue from the fifth passage of the nasopharyngeal secretions of Case 26. ${ }^{1}$ After waiting for 48 hours, during which time no change occurred in the blood or temperature, the rabbit was killed. The lungs appeared normal.

In three other similar experiments the rabbits were observed for several days after the second injection, and remained normal. The interval between injections in this series was 11 days, 2, 7, and 8 weeks. The usual control animals, $\mathrm{B}$ and $\mathrm{C}$, demonstrated the activity of the injected materials in each test.

These cross-protection experiments establish the antigenic identity of Bacterium pneumosintes with the active agent derived from the nasopharyngeal secretions of patients in the early hours of epidemic influenza.

\section{SUMMARY AND CONCLUSION.}

The experiments described furnish additional evidence of the pathogenic character and the virtual identity of the various strains of the active agent derived from the nasopharyngeal secretions of influenzal patients with which the transmission experiments in rabbits have been carried out.

The active material has been shown to be of antigenic nature, so that rabbits are protected from the effects of a second inoculation. The experiments indicate also the antigenic identity of the various strains of the active agent with each other and with Bacterium pneumosintes.

Finally, the experiments show that the protection may persist for 14 months which is the longest period yet tested. 